\title{
AN EQUIVARIANT DESCRIPTION OF CERTAIN HOLOMORPHIC SYMPLECTIC VARIETIES
}

\author{
PETER CROOKS
}

(Received 30 August 2017; accepted 24 October 2017; first published online 20 February 2018)

\begin{abstract}
Varieties of the form $G \times S_{\text {reg, }}$, where $G$ is a complex semisimple group and $S_{\text {reg }}$ is a regular Slodowy slice in the Lie algebra of $G$, arise naturally in hyperkähler geometry, theoretical physics and the theory of abstract integrable systems. Crooks and Rayan ['Abstract integrable systems on hyperkähler manifolds arising from Slodowy slices', Math. Res. Let., to appear] use a Hamiltonian $G$-action to endow $G \times S_{\text {reg }}$ with a canonical abstract integrable system. To understand examples of abstract integrable systems arising from Hamiltonian $G$-actions, we consider a holomorphic symplectic variety $X$ carrying an abstract integrable system induced by a Hamiltonian $G$-action. Under certain hypotheses, we show that there must exist a $G$-equivariant variety isomorphism $X \cong G \times S_{\text {reg }}$.
\end{abstract}

2010 Mathematics subject classification: primary 14L30; secondary 51H30, 53D20.

Keywords and phrases: equivariant geometry, holomorphic symplectic geometry, Lie theory.

\section{Introduction}

1.1. Some preliminaries. We will work exclusively over $\mathbb{C}$, understanding it as implicitly present whenever a base field is needed. Let $G$ be a connected, simply connected semisimple linear algebraic group having rank equal to $\operatorname{rk}(G)$, Lie algebra denoted $\mathfrak{g}$ and adjoint representation denoted Ad $: G \rightarrow \mathrm{GL}(\mathfrak{g})$. Note that Ad induces the adjoint action of $G$ on $\mathfrak{g}$, whose orbits are called the adjoint orbits of $G$. We shall let $O(x) \subseteq \mathfrak{g}$ denote the adjoint orbit containing $x \in \mathfrak{g}$, that is,

$$
O(x):=\left\{\operatorname{Ad}_{g}(x): g \in G\right\} .
$$

The Killing form is Ad-invariant and nondegenerate, and therefore induces an isomorphism $\mathfrak{g} \cong \mathfrak{g}^{*}$ between the adjoint and coadjoint representations of $G$. We will often deal with moment maps for Hamiltonian $G$-actions, which by virtue of our isomorphism $\mathfrak{g} \cong \mathfrak{g}^{*}$ shall always be regarded as taking values in $\mathfrak{g}$.

Let ad $: \mathfrak{g} \rightarrow \mathfrak{g l}(\mathfrak{g})$ be the adjoint representation of $\mathfrak{g}$. An element $x \in \mathfrak{g}$ is called regular when the dimension of $\operatorname{ker}\left(\operatorname{ad}_{x}\right)$ coincides with $\operatorname{rk}(G)$, and we shall let $\mathfrak{g}_{\text {reg }} \subseteq \mathfrak{g}$

The Institute of Differential Geometry at Leibniz Universität Hannover supported this work through the author's postdoctoral fellowship.

(C) 2018 Australian Mathematical Publishing Association Inc. 
denote the open dense subvariety of all regular elements. This subvariety is invariant under the adjoint action, and as such is a union of certain adjoint orbits called the regular adjoint orbits. Equivalently, an adjoint orbit is regular if and only if its dimension is $\operatorname{dim}(G)-\operatorname{rk}(G)$.

Recall that $(\xi, h, \eta) \in \mathfrak{g}^{\oplus 3}$ is called an $\mathfrak{s l}_{2}(\mathbb{C})$-triple if the relations

$$
[\xi, \eta]=h, \quad[h, \xi]=2 \xi, \quad[h, \eta]=-2 \eta
$$

hold in $\mathfrak{g}$, and is called a regular $\mathfrak{s l}_{2}(\mathbb{C})$-triple when we also have $\xi, \eta \in \mathfrak{g}_{\text {reg. }}$. Take a regular $\mathfrak{s l}_{2}(\mathbb{C})$-triple $(\xi, h, \eta)$, fixed for the duration of this paper, and consider its associated Slodowy slice

$$
S_{\text {reg }}:=\xi+\operatorname{ker}\left(\operatorname{ad}_{\eta}\right):=\left\{\xi+x: x \in \operatorname{ker}\left(\operatorname{ad}_{\eta}\right)\right\} \subseteq \mathfrak{g} .
$$

This slice is a $\operatorname{rk}(G)$-dimensional affine-linear subspace of $\mathfrak{g}$ enjoying the following properties: $S_{\text {reg }} \subseteq \mathfrak{g}_{\text {reg }}$ and each regular adjoint orbit meets $S_{\text {reg }}$ in a unique point (see [6, Theorem 8]). Taken together, these two properties imply that

$$
\varphi: S_{\text {reg }} \rightarrow \mathfrak{g}_{\text {reg }} / G, \quad x \mapsto O(x)
$$

defines an isomorphism of algebraic varieties.

1.2. The main motivating example. The affine variety $G \times S_{\text {reg }}$ has received some attention in the research literature. Among other things, it is known to carry a distinguished hyperkähler manifold structure (see [2]), and it arises as an important object in Moore and Tachikawa's discussion of certain two-dimensional topological quantum field theories (see [8]). This variety and its properties will feature prominently in our paper. To elaborate on this, let us use the term holomorphic symplectic variety for a smooth algebraic variety $X$ endowed with a holomorphic symplectic form $\omega$. A left action of $G$ on $X$ shall then be called Hamiltonian if the action is algebraic, $\omega$ is $G$-invariant and there exists a moment map, that is, a $G$ equivariant smooth algebraic variety morphism $\mu: X \rightarrow \mathfrak{g}$ satisfying

$$
d(\langle\mu, \theta\rangle)=\iota_{\theta_{X}} \omega
$$

for all $\theta \in \mathfrak{g}$. Here, $G$-equivariance is with respect to the adjoint action of $G$ on $\mathfrak{g},\langle\cdot, \cdot\rangle$ is the Killing form on $\mathfrak{g}$ and $\theta_{X}$ denotes the fundamental vector field on $X$ associated to $\theta \in \mathfrak{g}$.

It turns out that $G \times S_{\text {reg }}$ is canonically a holomorphic symplectic variety, a consequence of its hyperkähler structure. Moreover, $G$ acts freely on $G \times S_{\text {reg }}$ via

$$
g \cdot(h, x):=\left(h g^{-1}, x\right), \quad g \in G,(h, x) \in G \times S_{\text {reg }} .
$$

This action is Hamiltonian with moment map

$$
\mu_{\text {reg }}: G \times S_{\text {reg }} \rightarrow \mathfrak{g}, \quad(g, x) \mapsto-\operatorname{Ad}_{g^{-1}}(x)
$$

(see [3, Proposition 5]) known to be a submersion (see [3, Proposition 6]). The connected components of the fibres of $\mu_{\text {reg }}$ are therefore the leaves of a holomorphic foliation $\mathcal{F}_{\text {reg }}$ of $G \times S_{\text {reg }}$, and it is easily seen that these leaves are $\operatorname{rk}(G)$-dimensional. The pair $\left(G \times S_{\text {reg }}, \mathcal{F}_{\text {reg }}\right)$ is actually an example of an abstract integrable system of rank equal to $\operatorname{rk}(G)$ (see [3, Theorem 13]), for which we have the following definition. 
Definition 1.1. Let $X$ be a holomorphic symplectic variety and $\mathcal{F}$ a holomorphic foliation of $X$ with $r$-dimensional leaves. Then $(X, \mathcal{F})$ is an abstract integrable system of rank $r$ if each $x \in X$ has an open neighbourhood $U$, together with leaf-wise constant holomorphic functions on $U$ whose Hamiltonian vector fields span $T \mathcal{F} \subseteq T X$ on $U$.

A few brief comments are in order. Definition 1.1 is just a holomorphic counterpart of [4, Definition 2.6], in which Fernandes et al. introduced the notion of an abstract noncommutative integrable system in the smooth category. Note that Definition 1.1 suppresses the term 'noncommutative', which is in keeping with [3, Definition 2]. Very roughly speaking, this notion aims to describe certain integrable systems in purely foliation-theoretic terms. We refer the reader to [4] for further details.

Let us return to the main discussion. Note that $\mathcal{F}_{\text {reg }}$ is a foliation whose leaves are the connected components of the fibres of a moment map. It is therefore natural to seek conditions under which a moment map will, analogously to $\mu_{\text {reg }}$ in the case of $\left(G \times S_{\text {reg }}, \mathcal{F}_{\text {reg }}\right)$, induce an abstract integrable system. To this end, we have the following result.

Theorem 1.2 [3, Theorem 14]. Let $X$ be a holomorphic symplectic variety equipped with a locally free Hamiltonian $G$-action admitting $\mu: X \rightarrow \mathfrak{g}$ as a moment map. Let $\mathcal{F}_{\mu}$ denote the holomorphic foliation of $X$ whose leaves are the connected components of the fibres of $\mu .{ }^{1}$ Then the pair $\left(X, \mathcal{F}_{\mu}\right)$ is an abstract integrable system if and only if $\operatorname{dim}(X)=\operatorname{dim}(G)+\operatorname{rk}(G)$ and $\mu(X) \subseteq \mathfrak{g}_{\mathrm{reg}}$, in which case $\operatorname{rk}(G)$ is the rank of the system.

1.3. Description of the main result. This paper is an attempt to (at least partially) understand the class of abstract integrable systems $\left(X, \mathcal{F}_{\mu}\right)$ that arise by satisfying the hypotheses of Theorem 1.2. More precisely, let $X$ be a holomorphic symplectic variety endowed with a Hamiltonian action of $G$ and moment map $\mu: X \rightarrow \mathfrak{g}$. We would like to better understand those cases in which all of the following conditions are satisfied:

(a) the $G$-action is locally free;

(b) $\operatorname{dim}(X)=\operatorname{dim}(G)+\operatorname{rk}(G)$; and

(c) $\mu(X) \subseteq \mathfrak{g}_{\text {reg }}$.

Our main result, Theorem 1.3, imposes some slightly more restrictive conditions, and then completely classifies $X$ up to a $G$-equivariant variety isomorphism.

THeOREM 1.3. Let $X$ be a holomorphic symplectic variety endowed with a Hamiltonian G-action and admitting $\mu: X \rightarrow \mathfrak{g}$ as a moment map. If:

(i) $X$ is affine;

(ii) the G-action is free;

(iii) $\operatorname{dim}(X)=\operatorname{dim}(G)+\operatorname{rk}(G)$;

\footnotetext{
${ }^{1}$ Since the $G$-action is locally free, $\mu$ is a submersion (see [1, Proposition III.2.3]) and all fibres have the same dimension.
} 
(iv) $\mu(X)=\mathfrak{g}_{\text {reg }}$; and

(v) $\mu^{-1}(O)$ is an irreducible subvariety of $X$ for all adjoint orbits $O \subseteq \mathfrak{g}$,

then there exists a $G$-equivariant variety isomorphism $X \cong G \times S_{\text {reg. }}$.

We shall devote Section 2 to the proof of this theorem. In the interim, let us make a few remarks about the hypotheses appearing in Theorem 1.3.

Remark 1.4. As one might expect, $X=G \times S_{\text {reg }}$, the Hamiltonian action (1.2) and the moment map $\mu=\mu_{\text {reg }}$ satisfy Conditions (i) $-(\mathrm{v})$. The first three of these conditions are immediately seen to hold, while the fourth is satisfied by virtue of [3, Proposition 6]. To verify Condition (v), let $O \subseteq \mathfrak{g}$ be an adjoint orbit. Since $\mu(X)=\mathfrak{g}_{\text {reg }}$, we must have $\mu^{-1}(O)=\emptyset$ whenever $O$ is not regular. If $O$ is regular, then the isomorphism (1.1) implies that $-O:=\{-x: x \in O\}$ meets $S_{\text {reg }}$ in a unique point $y$, and one can use (1.3) to check that

$$
\mu^{-1}(O)=G \times\{y\} \subseteq G \times S_{\text {reg }} .
$$

We thus see that $\mu^{-1}(O)$ is irreducible for all adjoint orbits $O \subseteq \mathfrak{g}$.

Remark 1.5. We require $X$ to be affine in order to use [7, Section III, Corollary 1] in the proof of Theorem 1.3. This result considers a reductive linear algebraic group $H$ acting algebraically on an affine variety $Y$ and is formulated as follows: the canonical morphism $Y \rightarrow Y / H$ is a principal $H$-bundle if and only if $H$ acts freely on $Y .{ }^{1}$ For a more situation-specific version of this result, assume that $Y$ is also irreducible. Additionally, let $Z$ be a normal variety and $f: Y \rightarrow Z$ a surjective morphism with the property that each fibre is a single $H$-orbit. One can then show that there exists a variety isomorphism $Y / H \stackrel{\cong}{\rightarrow} Z$ making the diagram

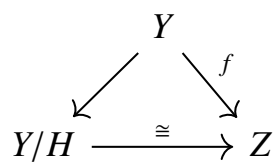

commute (see [11, Corollary 25.3.4 and Proposition 25.3.5]), so that [7, Section III, Corollary 1] takes the following form: $f$ is a principal $H$-bundle if and only if $H$ acts freely on $Y$. We will later apply this rephrased version of [7, Section III, Corollary 1] to argue that a particular map $X \rightarrow S_{\text {reg }}$ is a principal $G$-bundle, for which we must assume that $X$ (like $Y$ above) is affine.

Remark 1.6. Theorem 1.3 does not hold if one relaxes Condition (ii) to require only that the $G$-action be locally free. To see this, let $Z(G)$ denote the centre of $G$. The action (1.2) of $G$ on $G \times S_{\text {reg }}$ restricts to a $Z(G)$-action, which in turn commutes with the original $G$-action. In other words, $G \times S_{\text {reg }}$ carries a Hamiltonian action of $G \times Z(G)$. Now note that $Z(G)$ is a finite group, a consequence of having taken $G$ to be

\footnotetext{
${ }^{1}$ We shall always use the algebro-geometric notion of a principal bundle (see [7, Section I]), which is defined to be étale-locally trivial.
} 
semisimple. It follows that $\left(G \times S_{\text {reg }}\right) / Z(G)$ is the holomorphic symplectic quotient of $G \times S_{\text {reg }}$ by $Z(G)$ (see [5, Section 7.5] for details on holomorphic symplectic quotients). This quotient carries a residual Hamiltonian $G$-action whose moment map is obtained by letting $\mu_{\text {reg }}$ descend to the quotient $\left(G \times S_{\text {reg }}\right) / Z(G)$. An examination of (1.2) reveals that this quotient is $G$-equivariantly isomorphic to $(G / Z(G)) \times S_{\text {reg }}$, with $G$ acting on the first factor. The moment map on $(G / Z(G)) \times S_{\text {reg }}$ is given by

$$
\mu:(G / Z(G)) \times S_{\text {reg }} \rightarrow \mathfrak{g}, \quad([g], x) \mapsto-\operatorname{Ad}_{g^{-1}}(x) .
$$

One can now check that $X=(G / Z(G)) \times S_{\text {reg, }}$ its Hamiltonian $G$-action and the moment map $\mu$ satisfy Conditions (i), (iii), (iv) and (v), with the verification of (v) being almost identical to that given in Remark 1.4. However, note that $Z(G)$ is the $G$-stabiliser of each point in $X$. It follows that the $G$-action on $X$ is locally free but need not be free. Since $G$ acts freely on $G \times S_{\text {reg }}$, this means that $X$ need not be $G$ equivariantly isomorphic to $G \times S_{\text {reg }}$.

Remark 1.7. Let $O \subseteq \mathfrak{g}$ be an adjoint orbit. Condition (ii) implies that $\mu$ is a submersion (see [1, Proposition III.2.3]), so that $\mu^{-1}(O)$ is a smooth subvariety of $X$. In particular, Condition (v) holds if and only if $\mu^{-1}(O)$ is connected in the Zariski topology. This is in turn equivalent to the connectedness of $\mu^{-1}(O)$ in the complex analytic topology (see [9, Theorem 6.1]), which by virtue of $\mu$ being a submersion would hold if the fibres of $\mu$ were connected (also in the complex analytic topology). Hence, in the presence of Condition (ii), Condition (v) is weaker than $\mu$ being fibre-connected.

Condition (v) turns out to be strictly weaker than fibre-connectedness, even when one considers only those $X$ and $\mu$ satisfying (i)-(iv). Indeed, recall that (i)-(v) hold for the example considered in Remark 1.4. For the same example, it turns out that $\mu$ is not fibre-connected (see [3, Section 3.2]).

REMARK 1.8. Theorem 1.3 assumes that $\mu(X)=\mathfrak{g}_{\text {reg }}$ rather than the weaker condition $\mu(X) \subseteq \mathfrak{g}_{\text {reg }}$ discussed earlier. Indeed, the theorem no longer holds when one replaces the stronger condition with the weaker one. To see this, let $U$ be any affine open

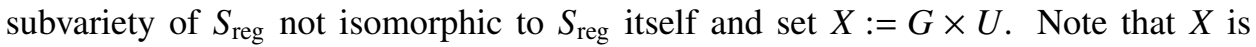
an open subvariety of $G \times S_{\text {reg }}$, so that the former inherits a holomorphic symplectic variety structure from the latter. Note also that $X$ is invariant under the $G$-action (1.2), which together with the previous sentence implies that (1.2) defines a Hamiltonian action on $X$. The moment map is $\left.\mu_{\text {reg }}\right|_{X}$.

It is not difficult to check that $X$, its Hamiltonian $G$-action and the moment map $\mu=\left.\mu_{\text {reg }}\right|_{X}$ satisfy Conditions (i)-(iii) in Theorem 1.3, and one can adapt the relevant part of Remark 1.4 to show that Condition (v) is also satisfied. Condition (iv) does not hold, however, as one can use (1.1), (1.3) and the fact that $U$ is a proper subvariety of $S_{\text {reg }}$ to show that $\mu(X)$ is a proper subset of $\mathfrak{g}_{\text {reg. }}$. The varieties $X$ and $G \times S_{\text {reg }}$ are also not $G$-equivariantly isomorphic, since $U$ being nonisomorphic to $S_{\text {reg }}$ precludes the quotients $X / G(\cong U)$ and $\left(G \times S_{\text {reg }}\right) / G\left(\cong S_{\text {reg }}\right)$ from being isomorphic. 


\section{Proof of the main result}

Assume that the hypotheses of Theorem 1.3 are satisfied and define $\bar{\mu}: X \rightarrow S_{\text {reg }}$ to be the following composite map:

$$
\bar{\mu}:=\left(X \stackrel{\mu}{\rightarrow} \mathfrak{g}_{\mathrm{reg}} \stackrel{\pi}{\rightarrow} \mathfrak{g}_{\mathrm{reg}} / G \stackrel{\varphi^{-1}}{\rightarrow} S_{\mathrm{reg}}\right),
$$

where $\pi$ is the quotient map and $\varphi$ is the isomorphism defined in (1.1). More concretely, $\bar{\mu}$ assigns to each $x \in X$ the unique point at which $S_{\text {reg }}$ intersects $O(\mu(x))$. It follows that

$$
\bar{\mu}^{-1}(y)=\mu^{-1}(O(y))
$$

for all $y \in S_{\text {reg. }}$.

Now fix a point $y \in S_{\text {reg. }}$. The fibre $\bar{\mu}^{-1}(y)$ is then nonempty, as Condition (iv) implies that $\bar{\mu}$ is surjective. Accordingly, we may choose a point $x \in \bar{\mu}^{-1}(y)$. At the same time, we can use (2.2) and the $G$-equivariance property of $\mu$ to conclude that $\bar{\mu}^{-1}(y)$ is a $G$-invariant subvariety of $X$. It follows that the $G$-orbit in $X$ through $x$, denoted $G \cdot x$, belongs to $\bar{\mu}^{-1}(y)$.

We will establish that $G \cdot x=\bar{\mu}^{-1}(y)$. To this end, note that (2.2) and Condition (v) show $\bar{\mu}^{-1}(y)$ to be irreducible. Proving $G \cdot x=\bar{\mu}^{-1}(y)$ therefore reduces to showing that $G \cdot x$ is closed and has dimension equal to that of $\bar{\mu}^{-1}(y)$. Accordingly, note that the closure of $G \cdot x$ is a union of the orbit itself and a (possibly empty) collection of strictly lower-dimensional $G$-orbits (see [11, Proposition 21.4.5]), while Condition (ii) implies that all $G$-orbits are $\operatorname{dim}(G)$-dimensional. These observations imply that $G \cdot x$ is closed and $\operatorname{dim}(G)$-dimensional. At the same time, Condition (ii) allows us to conclude that $\mu$ is a submersion (see [1, Proposition III.2.3]), giving rise to the following calculation:

$$
\begin{aligned}
\operatorname{dim}\left(\bar{\mu}^{-1}(y)\right)=\operatorname{dim}\left(\mu^{-1}(O(y))\right) & =\operatorname{dim}(X)-\operatorname{dim}\left(g_{\text {reg }}\right)+\operatorname{dim}(O(y)) \\
& =(\operatorname{dim}(G)+\operatorname{rk}(G))-\operatorname{dim}(G)+(\operatorname{dim}(G)-\operatorname{rk}(G)) \\
& =\operatorname{dim}(G),
\end{aligned}
$$

where we have used the fact that $\operatorname{dim}(O(y))=\operatorname{dim}(G)-\operatorname{rk}(G)$, a consequence of $O(y)$ being regular. Hence, $G \cdot x=\bar{\mu}^{-1}(y)$, as desired.

We have shown that each fibre of $\bar{\mu}$ is a single $G$-orbit, one of the hypotheses required in order to apply the version of [7, Section III, Corollary 1] discussed at the end of Remark 1.5. As for the other hypotheses, we know $G$ to be reductive, $X$ to be affine, $S_{\text {reg }}$ to be normal and $G$ to act freely on $X$. Only one hypothesis remains to be checked, namely that $X$ is irreducible. To this end, the following lemma will be useful.

\section{Lemma 2.1. The map $\bar{\mu}$ is a submersion.}

Proof. Recall the definition of $\bar{\mu}$ given in (2.1). Having noted that $\mu$ is a submersion, it will suffice to prove that $\varphi^{-1} \circ \pi$ is a submersion. To this end, suppose that $x \in \mathfrak{g}_{\text {reg }}$. 
By virtue of the isomorphism (1.1), there exist elements $g \in G$ and $y \in S_{\text {reg }}$ for which $x=\operatorname{Ad}_{g}(y)$. Now observe that

$$
\psi: S_{\text {reg }} \rightarrow \mathfrak{g}_{\text {reg }}, \quad z \mapsto \operatorname{Ad}_{g}(z)
$$

is a section of $\varphi^{-1} \circ \pi$ satisfying $\psi(y)=x$. It follows that $d_{x}\left(\varphi^{-1} \circ \pi\right) \circ d_{y} \psi$ must be the identity on $T_{y} S_{\text {reg }}$, where $d_{x}\left(\varphi^{-1} \circ \pi\right)$ and $d_{y} \psi$ are the differential of $\varphi^{-1} \circ \pi$ at $x$ and the differential of $\psi$ at $y$, respectively. This shows $d_{x}\left(\varphi^{-1} \circ \pi\right)$ to be surjective, and we conclude that $\varphi^{-1} \circ \pi$ is indeed a submersion.

REMARK 2.2. An alternative and perhaps more conceptual proof can be roughly sketched as follows. There exist $\operatorname{rk}(G)$ algebraically independent homogeneous generators of $\mathbb{C}[\mathfrak{g}]^{G}$, the algebra of Ad-invariant polynomials on $\mathfrak{g}$. One can assemble these polynomials into the components of a map $\mathfrak{g} \rightarrow \mathbb{C}^{\mathrm{rk}(G)}$, called the adjoint quotient, which is known to be a submersion when restricted to $\mathfrak{g}_{\text {reg }}$ (cf. [6, Theorem 9]). This restricted adjoint quotient and $\varphi^{-1} \circ \pi$ are related by composition with an isomorphism $\mathbb{C}^{\text {rk }(G)} \cong S_{\text {reg }}$, owing to the fact that $S_{\text {reg }}$ is a section of the adjoint quotient. It follows that $\varphi^{-1} \circ \pi$ is also a submersion, which, as noted in the proof above, is sufficient to conclude that $\bar{\mu}$ is a submersion.

Let us return to the proof of Theorem 1.3. We note that the fibres of $\bar{\mu}$ are connected in the complex analytic topology, as each fibre is a $G$-orbit. Together with Lemma 2.1, this implies that $X$ is itself connected in the complex analytic topology. In particular, $X$ is Zariski-connected. Since $X$ is smooth, this amounts to $X$ being irreducible.

By the discussion from the paragraph preceding Lemma 2.1, we may apply [7, Section III, Corollary 1] and conclude that $\bar{\mu}: X \rightarrow S_{\text {reg }}$ is a principal $G$-bundle. This bundle is trivial since the base $S_{\text {reg }}$ is affine space (see [10, Theorem C] or [12, Proposition 3.9]). In particular, there exists a $G$-equivariant variety isomorphism $X \cong G \times S_{\text {reg }}$.

\section{Acknowledgements}

The author is grateful to Roger Bielawski for asking the questions that ultimately prompted this work and to Steven Rayan for several fruitful discussions.

\section{References}

[1] M. Audin, Torus Actions on Symplectic Manifolds, revised edn, Progress in Mathematics, 93 (Birkhäuser, Basel, 2004).

[2] R. Bielawski, 'Hyperkähler structures and group actions', J. Lond. Math. Soc. (2) 55(2) (1997), 400-414.

[3] P. Crooks and S. Rayan, 'Abstract integrable systems on hyperkähler manifolds arising from Slodowy slices', Math. Res. Let., to appear.

[4] R. L. Fernandes, C. Laurent-Gengoux and P. Vanhaecke, 'Global action-angle variables for noncommutative integrable systems', J. Symplectic Geom., to appear.

[5] S. Kobayashi, Differential Geometry of Complex Vector Bundles, Publications of the Mathematical Society of Japan, vol. 15, Kanô Memorial Lectures, 5 (Princeton University Press, Princeton, NJ, 1987). 
[6] B. Kostant, 'Lie group representations on polynomial rings', Amer. J. Math. 85 (1963), 327-404.

[7] D. Luna, 'Slices étales', Mém. Soc. Math. France 33 (1973), 81-105.

[8] G. W. Moore and Y. Tachikawa, 'On 2d TQFTs whose values are holomorphic symplectic varieties', in String-Math 2011, Proceedings of Symposia in Pure Mathematics, 85 (American Mathematical Society, Providence, RI, 2012), 191-207.

[9] B. Osserman, 'Complex varieties and the analytic topology'. https://www.math.ucdavis.edu/ osserman/classes/248B-W12/notes/analytic.pdf.

[10] M. S. Raghunathan, 'Principal bundles on affine space', in: C. P. Ramanujam-a Tribute, Tata Institute of Fundamental Research Studies in Mathematics, 8 (Springer, New York, 1978), 187-206.

[11] P. Tauvel and R. W. T. Yu, Lie Algebras and Algebraic Groups, Springer Monographs in Mathematics (Springer, Berlin, 2005).

[12] M. Wendt, 'Rationally trivial torsors in $\mathbb{A}^{1}$-homotopy theory', J. K-Theory 7(3) (2011), 541-572.

PETER CROOKS, Institute of Differential Geometry,

Gottfried Wilhelm Leibniz Universität,

Hannover, Welfengarten 1, 30167 Hannover, Germany

e-mail: peter.crooks@math.uni-hannover.de 